PROCEEDINGS OF THE

AMERICAN MATHEMATICAL SOCIETY

Volume 127, Number 1, January 1999, Pages 289-295

S 0002-9939(99)04591-8

\title{
STRUCTURAL STABILITY ON BASINS FOR NUMERICAL METHODS
}

\author{
MING-CHIA LI
}

(Communicated by Mary Rees)

\begin{abstract}
In this paper, we show that a flow $\varphi$ with a hyperbolic compact attracting set is structurally stable on the basin of attraction with respect to numerical methods. The result is a generalized version of earlier results by Garay, Li, Pugh, and Shub. The proof relies heavily on the usual invariant manifold theory elaborated by Hirsch, Pugh, and Shub (1977), and by Robinson (1976).
\end{abstract}

\section{Statement of theorems}

Let $M$ be a smooth complete Riemannian manifold with a distance $d$ arising from the Riemannian metric and $\operatorname{Diff}(M)$ be the set of diffeomorphisms on $M$ with the strong topology and distance $d_{C^{1}}$. A flow is a map $\varphi: \mathbb{R} \times M \rightarrow M$ that satisfies the group property: $\varphi^{s}\left(\varphi^{t}(x)\right)=\varphi^{s+t}(x)$.

A set $\mathcal{A}$ is attracting for a flow $\varphi$ on $M$ if there is a neighborhood $U$ of $\mathcal{A}$ such that $\varphi^{T}(c l(U)) \subset \operatorname{int}(U)$ for some $T>0$ and $\mathcal{A}=\bigcap_{t \geq 0} \varphi^{t}(U)$. The basin of $\mathcal{A}$ is the set $B(\mathcal{A})=\left\{x \in M: \lim _{t \rightarrow \infty} d\left(\varphi^{t}(x), \mathcal{A}\right)=0\right\}$, where $d(y, \mathcal{A})=\min \{d(y, z): z \in \mathcal{A}\}$. An attracting set for a flow is closed and invariant (see [18]).

A compact invariant set $\mathcal{A}$ for a flow $\varphi$ on $M$ is hyperbolic if the restriction of the tangent bundle $T M$ of $M$ to $\mathcal{A}$ splits into three continuous subbundles, $T M \mid \mathcal{A}=\mathbb{E}^{u} \oplus \mathbb{E}^{s} \oplus \operatorname{Span}(X)$, invariant under the derivative of $\varphi^{t}, D \varphi^{t}$, such that $D \varphi^{t}$ expands $\mathbb{E}^{u}$ and $D \varphi^{t}$ contracts $\mathbb{E}^{s}$. Here $X$ is the vector field induced by the flow $\varphi$.

Definition 1. For $p \geq 1$, let $\varphi$ be a $C^{p+1}$ flow on $M$. A $C^{p+1}$ function $N$ : $\mathbb{R} \times M \rightarrow M$ is called a numerical method of order $p$ for $\varphi$ if there are positive constants $K$ and $h_{0}$ such that $d\left(\varphi^{h}(x), N^{h}(x)\right) \leq K h^{p+1}$, for all $h \in\left[0, h_{0}\right]$ and $x \in M$. Here $h$ stands for a stepsize of $N$. We denote the $i$-th iterate of $N^{h}(x)$ by $\left(N^{h}\right)^{i}(x)$.

Numerical methods arise from computer simulation and numerical approximation. For instance, both explicit and implicit Runge-Kutta methods are of order $p \geq 4$ (see [1]).

Received by the editors January 28, 1997 and, in revised form, May 6, 1997.

1991 Mathematics Subject Classification. Primary 58F10, 58F12, 65L20, 34D30, 34D 45.

Key words and phrases. Structural stability, dynamical systems, hyperbolic attracting set, basin of attraction, numerical method, Euler's method. 
It is well known that the time- $h$ map of the flow and the numerical method of stepsize $h$ are $C^{1}$ close exponentially in terms of $h$.

Lemma 1 ([7]). Let $N$ be a numerical method of order $p$ for a $C^{p+1}$ flow $\varphi$ on a compact manifold $M$. Then there is a constant $K_{1}$ such that $d_{C^{1}}\left(\varphi^{h}, N^{h}\right) \leq K_{1} h^{p}$ for all sufficiently small $h$. Moreover, given $T>0$, there is a constant $K_{2}$ such that $d_{C^{1}}\left(\varphi^{T},\left(N^{\frac{T}{n}}\right)^{n}\right) \leq K_{2} n^{1-p}$ for all large $n \in \mathbb{N}$.

Now we state our main result.

Theorem 1. Let $p \geq 2, \mathcal{A}_{\varphi}$ be a hyperbolic attracting set for a $C^{p+1}$ flow $\varphi$ on a compact manifold $M$, and $B\left(\mathcal{A}_{\varphi}\right)$ be the basin of $\mathcal{A}_{\varphi}$. Let $N$ be a numerical method of order $p$ for $\varphi$ and $T>0$ be given. If $n$ is sufficiently large, then there is a homeomorphism $H_{n}$ from $B\left(\mathcal{A}_{\varphi}\right)$ to its image and a continuous function $\tau_{n}$ : $B\left(\mathcal{A}_{\varphi}\right) \rightarrow \mathbb{R}$ such that for all $x \in B\left(\mathcal{A}_{\varphi}\right)$,

$$
H_{n} \circ \varphi^{\tau_{n}(x)}(x)=\left(N^{\frac{T}{n}}\right)^{n} \circ H_{n}(x) .
$$

Theorem 1 does not work for the Euler method because it is of order $p=1$ (see [1]). We discuss this case separately. In order to describe the Euler method on an abstract manifold, we need a local chart. As in [19], let $\Psi_{x}: U_{x} \rightarrow M$ be a function on a neighborhood $U_{x}$ of 0 in $T_{x} M$ such that $\Psi_{x}(0)=x, D \Psi_{x}(0)=i d_{T_{x} M}$, and $\Psi: U \rightarrow M$ defined by $(x, v) \rightarrow \Psi_{x}(v)$ is smooth with Lipschitz first and second derivative which are bounded along $M$.

Definition 2. Let $X$ be a vector field on $M$ and $\varphi$ be the flow of the differential equation $\dot{x}=X(x)$. For $h>0$ small, let $E^{h}(x)=\Psi_{x}(h X(x))$. We call $E^{h}$ the Euler method of stepsize $h$ for $\varphi$.

This agrees with the usual definition in Euclidean space where $\Psi_{x}(X(x))=$ $x+X(x)$ and $E^{h}(x)=x+h X(x)$.

Fortunately, we also have the $C^{1}$ closeness between the time- $h$ map of the flow and the Euler method of stepsize $h$. The following lemma was given by Shub [19].

Lemma 2. If $X$ is a $C^{2}$ bounded vector field on $M, \varphi$ is the flow of the differential equation $\dot{x}=X(x)$, and $E$ is the Euler method for $\varphi$. Then for all sufficiently small $h$, there is a positive constant $K_{1}$ such that $d_{C^{1}}\left(\varphi^{h}, E^{h}\right) \leq K_{1} h^{2}$. Moreover, given $T>0$, there is a positive constant $K_{2}$ such that $d_{C^{1}}\left(\varphi^{T},\left(E^{\frac{T}{n}}\right)^{n}\right) \leq K_{2} n^{-1}$ for all large $n \in \mathbb{N}$.

Omitting the same argument as in the proof of Theorem 1, we only state the result for the Euler method.

Theorem 2. Let $X$ be a $C^{2}$ vector field on a compact manifold $M$ such that the differential equation $\dot{x}=X(x)$ induces a flow $\varphi$ with a hyperbolic attracting set $\mathcal{A}_{\varphi}$. Let $E^{h}$ be the Euler method with stepsize $h$ for $\varphi$ and $T>0$ be given. Then for all sufficiently large $n$, there is a homeomorphism $H_{h}$ from $B\left(\mathcal{A}_{\varphi}\right)$ to its image and a continuous function $\tau_{n}: B\left(\mathcal{A}_{\varphi}\right) \rightarrow \mathbb{R}$ such that for all $x \in B\left(\mathcal{A}_{\varphi}\right)$,

$$
H_{n} \circ \varphi^{\tau_{n}(x)}(x)=\left(E^{\frac{T}{n}}\right)^{n} \circ H_{n}(x) .
$$

Our theorems fit well in a list of results on stability for numerical methods. Garay [8] asserted that the restriction of $\varphi$ to $B\left(\mathcal{A}_{\varphi}\right) \backslash \mathcal{A}_{\varphi}, \varphi \mid B\left(\mathcal{A}_{\varphi}\right) \backslash \mathcal{A}_{\varphi}$, is structurally stable under discretization. Beyn [2] proved that hyperbolic fixed points persist under numerical methods and the whole saddle-point structure in a neighborhood 
of a hyperbolic fixed point is preserved. Garay [6] showed that on a neighborhood of a hyperbolic fixed point, the time- $h$ map of the flow and a numerical method of stepsize $h$ can be conjugated by a local homeomorphism. Fečkan [5] proved the very same result in the case of the Euler method. See also [9] and [13] for structural stability of Morse-Smale gradient-like flows under numerics. Pugh and Shub [14] showed that a hyperbolic periodic orbit persists as an invariant embedded circle under solution schemes. Special cases were discussed in [2], [3] and [4]. See [14] and [7] for the persistence of normally hyperbolic invariant manifold and also [12] and [11] for the persistence of attractors.

\section{Proof of Theorem 1}

We closely follow the proofs of abstract invariant manifold theorems in [10] and [17]. We first outline the proof and then present the details.

We want to construct the conjugacy $H_{n}$ on the basin of attraction $B\left(\mathcal{A}_{\varphi}\right)$. The usual approach is to consider a $C^{1}$ bundle map $F$ defined on a fixed smooth normal bundle $\eta$ of the flow $\varphi$ contained in $T M$ and then to construct unstable manifolds for $F$ of the zero section of $\eta \mid U$, where $U$ is a small neighborhood of $\mathcal{A}_{\varphi}$. Following the methods in [10] and [17], we consider trial unstable manifolds which are graphs of sections $\sigma: \eta^{u}(r) \mid U \rightarrow \eta$, where $\eta \mid U=\eta^{u} \oplus \eta^{s}$ is an almost invariant splitting and $\eta^{u}(r)$ is the disk bundle of radius $r$. The graph transform by $F, F_{\#}(\sigma)$, is defined over $\varphi^{T}(U)$ in such a way that $F(\operatorname{image}(\sigma)) \supset \operatorname{image}\left(F_{\#}(\sigma)\right)$. We need to extend $F_{\#}(\sigma)$ back over a fundamental domain $V=U \backslash \varphi^{T}(U)$. We first construct a section over $V, \sigma_{0}: \eta^{u}(r) \mid V \rightarrow \eta$, whose graph is an invariant manifold for $F$ of the zero section. We then only consider sections $\sigma$ that agree with $\sigma_{0}$ over $V$, and extend $F_{\#}(\sigma)$ to $V$ by means of $\sigma_{0}$. By results of [10] and [17], we get that the graph transform $F_{\#}$ is a contraction on a space of sections. Hence there is a unique fixed point $\sigma^{u \varphi}: \eta^{u}(r) \mid U \rightarrow \eta$ such that $F_{\#}\left(\sigma^{u \varphi}\right)=\sigma^{u \varphi}$, and $\sigma^{u \varphi}=\sigma_{0}$ over $V$. The unstable manifolds for $F$ of the zero section of $\eta \mid U, Z^{u \varphi}(x)=\operatorname{image}\left(\sigma_{x}^{u \varphi}\right)$, are contained in $\eta$. We get what is called in [17] a family of unstable disks for $\varphi^{T}$, $\left\{Z^{u \varphi}(x): x \in U\right\}$.

To prove structural stability, for $\left(N^{\frac{T}{n}}\right)^{n}$ near $\varphi^{T}$ we consider a bundle map $G_{n}$ is $C^{1}$ near $F$. Again, we get families of stable and unstable disks for $G_{n}$ that are continuous and near those for $F$. Their intersection gives a section $v_{n}: U \rightarrow \eta$ that is continuous. Then $H_{n} \equiv \exp v_{n}$ is a semiconjugacy of $\varphi^{T}$ and $\left(N^{\frac{T}{n}}\right)^{n}$. By the expansiveness of $\varphi$ on $\mathcal{A}_{\varphi}$ and the way we construct $\sigma_{0}$ over $V$, we can show $H_{n}$ is one-to-one on $B\left(\mathcal{A}_{\varphi}\right)$.

We divide the proof into several steps.

Step 1: Preliminary setup.

Because $\mathcal{A}_{\varphi}$ is hyperbolic, the tangent bundle of $M$ along $\mathcal{A}_{\varphi}$ splits as the sum of three bundles $T M \mid \mathcal{A}_{\varphi}=\mathbb{E}^{u} \oplus \mathbb{E}^{s} \oplus \operatorname{Span}(X)$, where $X(x)=\left.\frac{d \varphi^{t}}{d t}(x)\right|_{t=0}$ is the tangent vector field for $\varphi$. Let $U_{0}$ be a small neighborhood of $\mathcal{A}_{\varphi}$. We want the normal bundle $\eta$ of $\varphi$ to be smooth. It is no loss of generality to make a convenient choice of $\eta$ : Let $\eta^{u}$ and $\eta^{s}$ be smooth subbundles of $T M \mid U_{0}$ with approximating $\mathbb{E}^{u}$ and $\mathbb{E}^{s}$ so that $T M \mid U_{0}=\eta^{u} \oplus \eta^{s} \oplus \operatorname{Span}(X)$, and choose $\eta=\eta^{u} \oplus \eta^{s}$. To make the analysis easier, we assume that the bundles $\eta^{u}$ and $\eta^{s}$ can be trivialized, i.e., for $\delta=u, s$, we assume there exists a $C^{1}$ bundle $B^{\delta}$ over $U_{0}$ such that $\eta^{\delta} \oplus B^{\delta}=U_{0} \times Y^{\delta}$, where $Y^{\delta}$ is a Banach space, and the Finsler (continuous norm) on $\eta^{\delta}$ is induced 


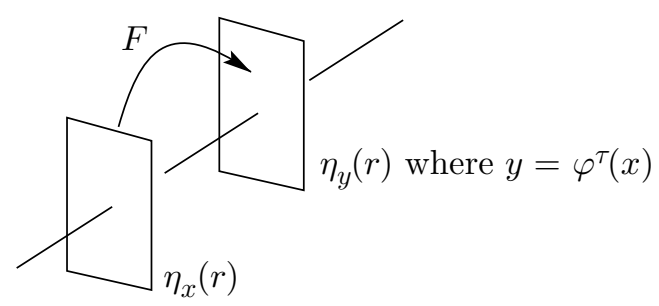

Figure 1

from the norm on $Y^{\delta}$. Let $\eta^{\delta}(r)=\left\{v \in \eta^{\delta}:|v| \leq r\right\}, \delta=u, s$, be the $r$ disk bundles and $\eta(r)=\eta^{u}(r) \oplus \eta^{s}(r)$.

To get a grip on the space of sections, we need to define a section's slope. If $\sigma: \eta^{u}(r) \rightarrow \eta$ is a section, then the slope of $\sigma$ at $v_{x} \in \eta^{u}(r)$ is

$$
\limsup _{v_{y} \rightarrow v_{x}} \frac{\left|s\left(v_{x}\right)-s\left(v_{y}\right)\right|_{Y^{s}}}{d_{u}\left(v_{x}, v_{y}\right)}
$$

where $\sigma\left(v_{x}\right)=\left(v_{x}, s\left(v_{x}\right)\right) \in \eta^{u} \times Y^{s},|\cdot|_{Y^{s}}$ is the norm on $Y^{s}$, and $d_{u}$ is the Finsler metric on $\eta^{u}$. Let $\Sigma(1, r)=\left\{\right.$ section $\sigma: \eta^{u}(r) \rightarrow \eta(r)$ such that slope $(\sigma) \leq 1$ \}. Putting the $C^{0}$ sup norm on $\Sigma(1, r)$ makes it a complete metric space as usual. Let $\pi^{u}: \eta \rightarrow \eta^{u}$ be a projection along $\eta^{s}$ and $\pi^{s}: \eta \rightarrow \eta^{s}$ be a projection along $\eta^{u}$.

Step 2: Definition of bundle map.

We use the concept of laminations in [10] to define a bundle map $F$ on $\eta$. For $f$ near $\varphi^{T}$ in the $C^{1}$ topology, let $\Theta\left(\tau, v_{x}, f\right)=\exp _{y}^{-1} \circ f \circ \exp v_{x}$ where $y=\varphi^{\tau}(x)$. There is a neighborhood $U_{1} \subset U_{0}$ of $\mathcal{A}_{\varphi}$, a constant $r_{1}>0$, a neighborhood $\mathcal{U}$ of $\varphi^{T}$ in $\operatorname{Diff}(M)$, and a continuous function $\tau:\left.\eta\left(r_{1}\right)\right|_{U_{1}} \times \mathcal{U} \rightarrow \mathbb{R}$ such that for all $x \in U_{1}, v_{x} \in \eta_{x}\left(r_{1}\right)$, and $f \in \mathcal{U}$,

$$
\Theta\left(\tau\left(v_{x}, f\right), v_{x}, f\right) \in \eta_{\varphi^{\tau\left(v_{x}, f\right)}(x)}\left(r_{1}\right) .
$$

Here $\tau$ stands for a reparameterization of $\varphi$. See page 95 of [10] and also [16]. Define a bundle map $F$ by

$$
F\left(v_{x}\right) \equiv \Theta\left(\tau\left(v_{x}, \varphi^{T}\right), v_{x}, \varphi^{T}\right)=\exp _{\varphi^{\tau}(x)}^{-1} \circ \varphi^{T} \circ \exp v_{x} .
$$

Then $F$ is a $C^{1}$ bundle map on $\eta\left(r_{1}\right)$. See Figure 1.

Step 3: Definition of graph transform.

To define the graph transform of $F$, we need an inverse of $\pi^{u} \circ F \circ \sigma$ for $\sigma \in \Sigma(1, r)$. As in page 102 of [10], one can take $r_{2} \leq r_{1}$ small so that for $\sigma \in \Sigma\left(1, r_{2}\right)$ and $x \in \mathcal{A}_{\varphi}, \pi^{u} \circ F \circ \sigma:\left.\left.\eta^{u}\left(r_{2}\right)\right|_{O(x)} \rightarrow \eta^{u}\right|_{O(x)}$ is invertible, where $O(x)$ is the trajectory of $x$ under the flow $\varphi$. Let $U_{2} \subset U_{1}$ be a small neighborhood of $\mathcal{A}_{\varphi}$ such that $\pi^{u} \circ F \circ \sigma$ is invertible on $\left.\eta^{u}\left(r_{2}\right)\right|_{U_{2}}$ and has an inverse $g$ defined on $\left.\eta^{u}\left(r_{2}\right)\right|_{\varphi^{T}\left(U_{2}\right)}$, $g:\left.\eta^{u}\left(r_{2}\right)\right|_{\varphi^{T}\left(U_{2}\right)} \rightarrow \eta^{u}\left(r_{2}\right)$.

In order to construct the conjugacy not only on the attractor but also on the basin of attraction, we adapt the method of Robinson [17], see also [15]. Let $U \subset U_{2}$ be a small neighborhood of $\mathcal{A}_{\varphi}$ so that $V=\operatorname{closure}\left(U \backslash \varphi^{T}(U)\right)$ is a proper fundamental domain. Here $\varphi^{T}(U) \subset U$ because $\mathcal{A}_{\varphi}$ is an attracting set. Let $\beta$ be a bump function that equals zero near the exterior boundary of $V$, or $\partial U$, and equals 1 near the interior boundary of $V$, or $\partial \varphi^{T}(U)$. Let $\sigma_{1}: \eta^{u}\left(r_{2}\right) \rightarrow \eta$ be defined by $\sigma_{1}(v)=v$, i.e. $\pi^{s} \sigma_{1}(v)=0$, and $\sigma_{2}=F \circ \sigma_{1} \circ g$. Define $\sigma_{0}: \eta^{u} \rightarrow \eta$ by $\pi^{s} \sigma_{0}\left(v_{x}\right)=\beta(x) \pi^{s} \sigma_{2}\left(v_{x}\right)$. 


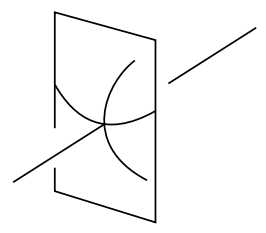

$\mathrm{a}$

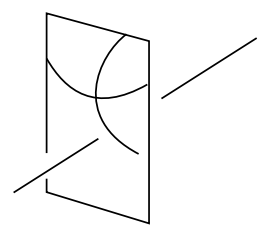

$\mathrm{b}$

Figure 2

Now we define a graph transform $F_{\#}$ of $F$ over $U$ by

$$
F_{\#}(\sigma)_{x}= \begin{cases}F \circ \sigma \circ g_{x} & \text { for } x \in \varphi^{T}(U), \\ \sigma_{0 x} & \text { for } x \in V .\end{cases}
$$

Let $\Sigma\left(1, r, \sigma_{0}\right)=\left\{\right.$ section $\sigma: \eta^{u}(r) \rightarrow \eta(r)$ such that $\sigma=\sigma_{0}$ on the domain of $\sigma_{0}$ and slope $(\sigma) \leq 1\}$. By Theorem 6.1 of [10] and Theorem 3.1 of [17], we get that $F_{\#}$ is a contraction on $\Sigma\left(1, r_{2}, \sigma_{0}\right)$ and has a unique fixed point $\sigma^{u \varphi} \in \Sigma\left(1, r_{2}, \sigma_{0}\right)$.

Step 4: Construction of conjugacy and reparameterization.

In order to prove structural stability, we do the same construction for $\left(N^{\frac{T}{n}}\right)^{n}$. By Lemma 1, we have $\left(N^{\frac{T}{n}}\right)^{n} \rightarrow \varphi^{T}$ in the $C^{1}$ topology as $n \rightarrow \infty$. Take $n$ sufficiently large so that $\left(N^{\frac{T}{n}}\right)^{n} \in \mathcal{U}$, the neighborhood of $\varphi^{T}$ in $\operatorname{Diff}(M)$ where $\Theta$ is well-defined. Let

$$
G_{n}\left(v_{x}\right) \equiv \Theta\left(\tau\left(v_{x},\left(N^{\frac{T}{n}}\right)^{n}\right), v_{x},\left(N^{\frac{T}{n}}\right)^{n}\right)=\exp _{\varphi^{\tau}(x)}^{-1} \circ\left(N^{\frac{T}{n}}\right)^{n} \circ \exp v_{x} .
$$

Then $G_{n}$ is $C^{1}$ bundle map on $\eta\left(r_{1}\right)$ and $C^{1}$ close to $F$. We define $\sigma_{n 0}^{N}$ over $V$ by $\sigma_{n 0}^{N}\left(v_{x}\right)=\beta(x)\left(N^{\frac{T}{n}}\right)^{n} \circ \sigma_{1} \circ g\left(v_{x}\right)$. Because $\left(N^{\frac{T}{n}}\right)^{n}$ is $C^{1}$ near $\varphi^{T}, \sigma_{n 0}^{N}$ is $C^{1}$ near $\sigma_{0}$. By the permanence results in Theorem 3.1 of [17] and Theorem 6.1 of [10], the graph transform of $G_{n}, G_{n \#}$, is $C^{1}$ close to $F_{\#}$, carries $\Sigma\left(1, r_{2}, \sigma_{n 0}^{N}\right)$ into itself, and is a contraction. Therefore, $G_{n \#}$ has a unique fixed point $\sigma_{n}^{u N} \in \Sigma\left(1, r_{2}, \sigma_{n 0}^{N}\right)$.

Because $\varphi^{-T}$ is overflowing on $U$, without defining $\sigma_{0}$ on a fundamental domain, we can construct $\sigma^{s \varphi}$ and $\sigma_{n}^{s N}$. The disks $Z^{u}(x, \varphi)=\operatorname{image}\left(\sigma_{x}^{u \varphi}\right)$ and $Z^{s}(x, \varphi)=$ image $\left(\sigma_{x}^{s \varphi}\right)$ intersect transversally at the zero vector, see Figure 2a. The disks $Z_{n}^{u}(x, N)=\operatorname{image}\left(\sigma_{n x}^{u N}\right)$ and $Z_{n}^{s}(x, N)=\operatorname{image}\left(\sigma_{n x}^{s N}\right)$ intersect transversally in each fiber at a unique point, $v_{n}(x)$, see Figure $2 \mathrm{~b}$. Then $v_{n}: U \rightarrow \eta\left(r_{2}\right)$ is a continuous section and $G_{n \#} v_{n}=v_{n}$. We extend $v_{n}$ to the basin of attraction, $B\left(\mathcal{A}_{\varphi}\right)$.

We now define the conjugacy and the reparameterization. Let $H_{n}(x)=\exp v_{n}(x)$ and $\tau_{n}(x)=\tau\left(v_{n}(x),\left(N^{\frac{T}{n}}\right)^{n}\right)$ for all $x \in B\left(\mathcal{A}_{\varphi}\right)$. Then $H_{n}: B\left(\mathcal{A}_{\varphi}\right) \rightarrow M$ and $\tau_{n}: B\left(\mathcal{A}_{\varphi}\right) \rightarrow \mathbb{R}$ are continuous. Moreover $v_{n} \circ \varphi^{\tau_{n}(x)}(x)=\exp _{\varphi^{\tau_{n}(x)}(x)}^{-1} \circ\left(N^{\frac{T}{n}}\right)^{n} \circ$ $\exp v_{n}(x)$ and so $H_{n} \circ \varphi^{\tau_{n}(x)}(x)=\left(N^{\frac{T}{n}}\right)^{n} \circ H_{n}(x)$. It remains to show that $H_{n}$ is one-to-one.

Step 5: Prove $H_{n}$ is one-to-one.

Assume that $H_{n}(x)=H_{n}(y)$. Then $H_{n} \circ \varphi^{\tau_{n}(x)}(x)=\left(N^{\frac{T}{n}}\right)^{n} \circ H_{n}(x)=\left(N^{\frac{T}{n}}\right)^{n} \circ$ $H_{n}(y)=H_{n} \circ \varphi^{\tau_{n}(y)}(y)$. There exist $\left\{x_{i}\right\}$ and $\left\{y_{i}\right\}$ lying on the flow trajectories of $x$ and $y$, respectively, so that $H_{n}\left(x_{i}\right)=\left(\left(N^{\frac{T}{n}}\right)^{n}\right)^{i}\left(H_{n}(x)\right)=\left(\left(N^{\frac{T}{n}}\right)^{n}\right)^{i}\left(H_{n}(y)\right)=$ $H_{n}\left(y_{i}\right)$ as long as $x_{i}, y_{i} \in B\left(\mathcal{A}_{\varphi}\right)$. Therefore $\exp v_{n}\left(x_{i}\right)=\exp v_{n}\left(y_{i}\right)$ and $d\left(x_{i}, y_{i}\right) \leq$ 
$2 r_{2}$ for all $i \geq 0$. Suppose $x \in \mathcal{A}_{\varphi}$. By the flow expansiveness of $\varphi$ at $\mathcal{A}_{\varphi}$ (see [18]), the points $x$ and $y$ must lie in the same flow trajectory. But transversal disks are disjoint for two nearby points on the same trajectory. Thus $x=y$. This proves $H_{n}$ is one-to-one on $\mathcal{A}_{\varphi}$.

Suppose $x \notin \mathcal{A}_{\varphi}$, then take $i_{0}$ so that $x_{i_{0}} \in V$ and $y_{i_{0}}$ is near $V$. It is still true that $\left\{y_{i}: i \geq 0\right\}$ stays near the forward trajectories of $\left\{x_{i_{0}}\right\}$ under $\varphi^{t}$, and so $y_{i_{0}} \in$ $W^{s}\left(O\left(x_{i_{0}}\right), \varphi^{t}\right)$, the stable manifold of the orbit of $x_{i_{0}}$. On $V$, the unstable disks $Z^{u}(z, N)$ are uniformly $C^{1}$ and transverse to the stable direction $W^{s}\left(O\left(x_{i_{0}}\right), \varphi^{t}\right)$. Thus the unstable disks form a tubular neighborhood of $W^{s}\left(O\left(x_{i_{0}}\right), \varphi^{t}\right)$. On the other hand, the fact that $\exp v_{n}\left(x_{i_{0}}\right)=\exp v_{n}\left(y_{i_{0}}\right)$ gives us $v_{n}\left(x_{i_{0}}\right) \in Z^{u}\left(x_{i_{0}}, N\right)$ and $v_{n}\left(y_{i_{0}}\right) \in Z^{u}\left(y_{i_{0}}, N\right)$, and so these two unstable disks intersect. Therefore, $x_{i_{0}}=y_{i_{0}}$ and we get that $x$ and $y$ are in the same flow trajectory. Again $x=y$ as above. This proves that $H_{n}$ is one-to-one on $B\left(\mathcal{A}_{\varphi}\right)$.

\section{ACKNOWLEDGMENT}

The author is indebted to Professor Clark Robinson of Northwestern University for helpful conversations.

\section{REFERENCES}

1. K. E. Atkinson, An Introduction to Numerical Analysis, second ed., John Wiley \& Sons, New York, 1989. MR 90m:65001

2. W. J. Beyn, On the numerical approximation of phase portraits near stationary points, SIAM J. Numer. Anal. 24 (1987), no. 5, 1095-1113. MR 88j:65143

3. M. Braun and J. Hershenov, Periodic solutions of finite difference equations, Quarterly of Applied Math. 35 (1977), 139-147. MR 58:23212

4. T. Eirola, Invariant curves of one-step methods, BIT (1988), no. 28, 113-122. MR 89g:65109

5. M. Fečkan, The relation between a flow and its discretization, Math. Slovaca 42 (1992), 123127. MR 93f:34071

6. B. M. Garay, Discretization and some qualitative properties of ordinary differential equations about equilibria, Acta Math. Univ. Commeniznae 62 (1993), 249-275. MR 95i:34079

7. _ Discretization and normal hyperbolicity, Z. Angew. Math. Mech. 74 (1994), T662T663.

8. The discretized flow on domains of attraction: a structural stability result, (1997), IMA J. Numer. Anal., to appear.

9. methods, Numer. Math. 72 (1996), 449-479. MR 97d:34007

10. M. Hirsch, C. Pugh, and M. Shub, Invariant Manifolds, Lecture Notes in Math, vol. 583, Springer-Verlag, New York, 1977. MR 58:18595

11. A. R. Humphries and A. M. Stuart, Runge-Kutta methods for dissipative and gradient dynamical systems, SIAM J. Numer. Anal. 31 (1994), 1452-1485. MR 95m:65116

12. P. E. Kloeden and J. Lorenz, Stable attracting sets in dynamical systems and their one-step discretizations, SIAM J. Numer. Anal. 23 (1986), 986-995. MR 87k:34074

13. M.-C. Li, Structural stability of Morse-Smale gradient-like flows under discretizations, SIAM J. Mathematical Analysis 28 (1997), 381-388. CMP 97:08

14. C. Pugh and M. Shub, $C^{r}$ stability of periodic solutions and solution schemes, Appl. Math. Letters 1 (1988), 281-285. MR 89m:58117

15. C. Robinson, The geometry of the structural stability proof using unstable disks, Bol. Soc. Brasil. Math. 6 (1975), 129-144. MR 58:13190

16. _ Structural stability of $C^{1}$ flows, Lectures Notes in Math., vol. 468, Springer-Verlag, Berlin, 1975, pp. 262-277. MR 58:31251

17. _ Structural stability of $C^{1}$ diffeomorphisms, J. Differential Equations 22 (1976), 28-73. MR 57:14051 
18. _ Dynamical Systems: Stability, Symbolic Dynamics, and Chaos, CRC Press, Boca Raton, FL, 1995. MR 97e:58064

19. M. Shub, Some remarks on dynamical systems and numerical analysis, Dynamical Systems and Partial Differential Equations, Proceeding of the VII Elam, Equinoccio, Universidad Simon Bolivar, Caracas, 1986, pp. 69-91. MR 88j:58065

Department of Mathematics, National Changhua University of Education, Changhua 500, TAIWAN

E-mail address: mcli@math.ncue.edu.tw 